\title{
Connection between Feynman integrals having different values of the space-time dimension
}

\author{
O. V. Tarasov* \\ Deutsches Electronen-Synchrotron DESY, Institut für Hochenergiephysik IfH, Zeuthen, Platanenallee 6, D-15738 Zeuthen, Germany
}

(Received 5 June 1996)

\begin{abstract}
A systematic algorithm for obtaining recurrence relations for dimensionally regularized Feynman integrals with respect to the space-time dimension $d$ is proposed. The relation between $d$ - and $(d-2)$-dimensional integrals is given in terms of a differential operator for which an explicit formula can be obtained for each Feynman diagram. We show how the method works for one-, two-, and three-loop integrals. The new recurrence relations with respect to $d$ are complementary to the recurrence relations which derive from the method of integration by parts. We find that the problem of the irreducible numerators in Feynman integrals can be naturally solved in the framework of the proposed generalized recurrence relations. [S0556-2821(96)04522-5]

PACS number(s): 11.10.Gh, 11.10.Kk, 12.38.Bx
\end{abstract}

\section{INTRODUCTION}

Many phenomena in high energy, solid state, and statistical physics can be described by quantum field theoretical models considered not only in four-dimensional space-time but also in two, three, and other space-time dimensions [1]. In many cases perturbation theory is the basic tool for calculating different physical quantities. The same Feynman diagrams occur in different models with different space-time dimension $d$. This parameter may be considered as a regularization parameter [2] different from the value of the physical space-time dimension. Usually one needs to set up a Laurent expansion of the diagram with respect to $\varepsilon=(2 l-d) / 2$, with $2 l$ being the dimension of the physical space-time in the problem under consideration. The coefficients of the expansion are different for different $l$. The most advanced methods were developed for the evaluation of $d=4-2 \varepsilon$ dimensional integrals. For example, the standard package MINCER [3] for calculating massless three-loop diagrams is now widely available and has been used in many multiloop calculations.

Another reason to consider $d$ different from its physical value was given in [4]. There, a new approach for obtaining nonperturbative information from a quantum field theory by expanding Green's functions as a series in powers of $d$ was proposed. In light of this investigation one can try to formulate approximate methods for calculating individual Feynman diagrams at some asymptotic values of $d$, for example, as $|d| \rightarrow \infty$ or as $|d| \rightarrow 0$.

One of the most powerful methods for evaluating Feynman diagrams is the method of integration by parts [5]. In this approach dimensionally regularized Feynman integrals are considered as functions of the exponents of the scalar propagators. Integration by parts gives relations connecting integrals with some exponents changed by \pm 1 , very similar to the relations for the contiguous hypergeometric functions. This is not a big surprise since it has been known for some time [6] that Feynman amplitudes belong to the class of hypergeometric functions. In many cases, integrals are just pro-

\footnotetext{
*On leave of absence from JINR, 141980 Dubna (Moscow Region), Russian Federation. Electronic address: tarasov@ifh.de
}

portional to the well-studied hypergeometric functions [7-9].

Using the recurrence relations, a variety of Feynman integrals can be reduced to the restricted set of so called "master integrals.' The proof of completeness for the set of recurrence relations obtained by integration by parts and the problem of a systematic algorithm how to use them for an arbitrary diagram are open questions. Any additional information in this respect may be useful for solving both problems.

In the present paper we propose a systematic formulation of the recurrence relations with respect to $d$. We shall show that these relations cannot be obtained by the method of integration by parts and therefore should be considered as an important addition to the Chetyrkin-Tkachov (CT) recurrence relations. As a concrete example, we demonstrate that $\mathrm{CT}$ recurrence relations with the recurrence relation with respect to $d$ compose all possible recurrence relations for the considered integral. New recurrence relations are not of purely academic interest. We demonstrate how one can calculate Feynman integrals using these relations. We also rederive some useful relations for one-loop diagrams with arbitrary number of external legs. The relations connecting integrals with different $d$ may be also useful for calculating integrals with $d \neq 4$.

We show how tensor integrals can be represented in terms of integrals with the changed $d$. This representation allows us to write Feynman integrals with irreducible numerators as a combination of scalar integrals having different values of $d$. Thus, the solution of the generalized system of recurrence relations automatically leads to the solution of the problem of irreducible numerators.

It turns out that the new recurrence relations are especially useful in the new method for the momentum expansion of the scalar Feynman integrals proposed in [10].

The paper is organized as follows. In Sec. II, we present the main ideas of our method. First, using the parametric representation we derive relation for arbitrary scalar integrals with different $d$. Then we show how tensor integrals can be expressed in terms of combinations of scalar integrals having different values of the space-time dimension. In the framework of this approach the solution to the problem of irreducible numerators in Feynman integrals is proposed. An algorithm for obtaining new generalized recurrence relations 
including integrals with different $d$ as well as different exponents of the propagators is developed.

In Sec. III, explicit relations connecting integrals with shifted $d$ for some one-, two-, and three-loop integrals are derived.

In Sec. IV, analogous relations for the one-loop integrals with an arbitrary number of external legs and arbitrary powers of propagators are given. We reproduced already known results for these integrals and obtained more general new ones.

In Sec. V, we demonstrate how one can calculate integrals explicitly by solving recurrence relations with respect to the dimension of space-time.

In Sec. VI, the new recurrence relations are used to calculate the $\varepsilon=(6-d) / 2$ expansion for the two-loop selfenergy diagrams from the expansion developed at $\varepsilon=(4-d) / 2$.

\section{RELATIONS FOR INTEGRALS WITH DIFFERENT $\boldsymbol{d}$}

The subject of our consideration will be dimensionally regularized scalar Feynman integrals. An arbitrary scalar $L$ loop integral can be written as

$$
G^{(d)}\left(\left\{s_{i}\right\},\left\{m_{s}^{2}\right\}\right)=\prod_{i=1}^{L} \int d^{d} k_{i} \prod_{j=1}^{N} P_{\frac{k_{j}}{\nu_{j}}, m_{j}},
$$

where

$$
P_{k, m}^{\nu}=\frac{1}{\left(k^{2}-m^{2}+i \epsilon\right)^{\nu}}, \quad \overline{k_{j}^{\mu}}=\sum_{n=1}^{L} \omega_{j n} k_{n}^{\mu}+\sum_{m=1}^{E} \eta_{j m} q_{m}^{\mu},
$$

$q_{m}$ are external momenta, $\left\{s_{i}\right\}$ is a set of scalar invariants formed from $q_{m}, N$ is the number of lines, $E$ is the number of external legs, and $\omega$ and $\eta$ are matrices of incidences of the diagram with the matrix elements being \pm 1 or 0 (see, for example, Ref. [11]).

To find the desired relation we shall use the parametric representation of the integral, which can be found in the literature $[11,12]$. For an arbitrary scalar Feynman integral in $d$-dimensional space-time we have

$$
\begin{aligned}
G^{(d)}\left(\left\{s_{i}\right\},\left\{m_{s}^{2}\right\}\right)= & i^{L}\left(\frac{\pi}{i}\right)^{d L / 2} \prod_{j=1}^{N} \frac{i^{-\nu_{j}}}{\Gamma\left(\nu_{j}\right)} \\
& \times \int_{0}^{\infty} \cdots \int_{0}^{\infty} \frac{d \alpha_{j} \alpha_{j}^{\nu_{j}-1}}{[D(\alpha)]^{d / 2}} \\
& \times \exp \left[i\left(\frac{Q\left(\left\{s_{i}\right\}, \alpha\right)}{D(\alpha)}-\sum_{l=1}^{N} \alpha_{l}\left(m_{l}^{2}-i \epsilon\right)\right)\right],
\end{aligned}
$$

where $D(\alpha)$ and $Q\left(\left\{s_{i}\right\}, \alpha\right)$ are homogeneous polynomials in $\alpha$ of degree $L$ and $L+1$, respectively. They can be represented as sums over trees and two-trees of the graph (see, for example, [13]):

$$
\begin{gathered}
D(\alpha)=\sum_{\substack{\text { over } \\
\text { trees }}}\left(\prod_{\substack{\text { over } \\
\text { chords }}} \cdots \alpha_{j} \cdots\right), \\
Q\left(\left\{s_{i}\right\}, \alpha\right)=\sum_{\substack{\text { over } \\
2 \text { trees chords }}} \prod_{\substack{\text { over } \\
\text { chor }}} \cdots \alpha_{j} \cdots\left(\sum_{\substack{\text { over comp. } \\
\text { of } 2 \text { tree }}} q\right)^{2} .
\end{gathered}
$$

These polynomials are characteristic functions of the topology of the diagram and of its subgraphs. Since $D$ and $Q$ will play an important role in the rest of the present paper, we remind the reader of the definitions of the trees and two-trees for connected diagrams. Any connected subdiagram of the diagram $G$ containing all the vertices of $G$ but is free of cycles (loops) is called a tree of $G$. Similarly, a two-tree is defined as any subdiagram of $G$ containing all the vertices of the original diagram, but is free of cycles, and consisting of exactly two connected components. Finally, a chord of a tree (two-tree) is defined as any line not belonging to this tree (two-tree).

The most illustrative will be graphical representation of $D$ and $Q$. As an example we present function $D(\alpha)$ for the two-loop propagator-type diagram which will be considered in Sec. VI. The graph itself and all possible trees are pictured in Fig. 1.

According to the above definition all lines which were removed from the graph to make a tree are chords of the tree. The contribution to $D(\alpha)$ from a tree will be product of $\alpha$ 's corresponding to its chords. Summing over all trees given in Fig. 1 we readily get

$$
\begin{aligned}
D(\alpha)= & \alpha_{1} \alpha_{5}+\alpha_{2} \alpha_{5}+\alpha_{3} \alpha_{5}+\alpha_{4} \alpha_{5}+\alpha_{1} \alpha_{2} \\
& +\alpha_{3} \alpha_{4}+\alpha_{2} \alpha_{3}+\alpha_{1} \alpha_{4} .
\end{aligned}
$$

The numbering of $\alpha$ 's corresponds to the numbering of lines in the figure. Other examples of $D$ and $Q$ for some particular diagrams will be given in the next sections.

In the case when the $\nu_{j}$ 's do not depend on $d$, one can see from Eq. (3) that $d$ enters the integrand in a rather simple way. Only the exponent of $D(\alpha)$ linearly depends on the dimension of space-time.

In order to find the relation between integrals in different dimensions $d$ we have to assume, at first, that all scalar propagators in Eq. (1) have different masses. Next, we introduce the polynomial differential operator

$$
D\left(\frac{\partial}{\partial m_{j}^{2}}\right)
$$
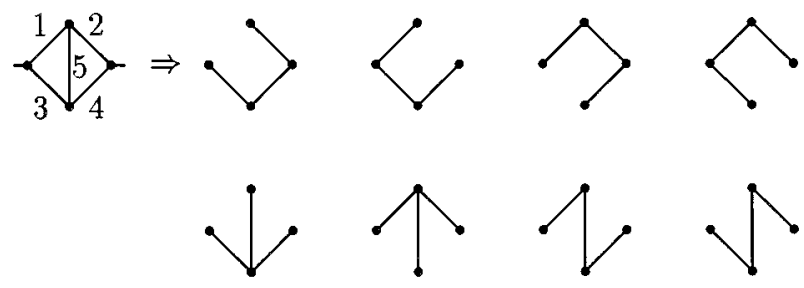

FIG. 1. Trees for the two-loop self-energy graph. 
which is obtained from $D(\alpha)$ by substituting $\alpha_{j} \rightarrow \partial_{j} \equiv \partial / \partial m_{j}^{2}$. The application of the operator $D(\partial)$ to the integral (3) gives $D(\alpha)$ in the numerator of the integrand:

$$
D(\partial) \exp \left(-i \sum \alpha_{l} m_{l}^{2}\right) \rightarrow D(\alpha)(-i)^{L} \exp \left(-i \sum \alpha_{l} m_{l}^{2}\right)
$$

The resulting integral is proportional to the same integral with $d$ changed to $d-2$ :

$$
G^{(d-2)}\left(\left\{s_{j}\right\},\left\{m_{s}^{2}\right\}\right)=\left(-\frac{1}{\pi}\right)^{L} D(\partial) G^{(d)}\left(\left\{s_{j}\right\},\left\{m_{s}^{2}\right\}\right) .
$$

After having performed the differentiation we identify masses with the one's of the original integral.

We may include tensor integrals into our consideration using standard methods $[11,13]$. To each line one introduces an auxiliary vector $a_{j}$ and then differentiates with respect to these vectors. The parametric representation for a tensor integral with products of $n_{j}$ vectors corresponding to the $j$ th line reads

$$
\begin{aligned}
\prod_{i=1}^{L} \int d^{d} k_{i} \prod_{j=1}^{N} P_{\bar{k}_{j}}^{\nu_{j}} \prod_{j} \prod_{l=1}^{n_{1}} \bar{k}_{1 \mu_{l}} \ldots \prod_{s=1}^{n_{N}} \bar{k}_{N \lambda_{s}}= & i^{L}\left(\frac{\pi}{i}\right)^{d L / 2} \prod_{j=1}^{N} \frac{i^{-\nu_{j}-n_{j}}}{\Gamma\left(\nu_{j}\right)} \prod_{r=1}^{n_{1}} \frac{\partial}{\partial a_{1 \mu_{r}}} \cdots \prod_{s=1}^{n_{N}} \frac{\partial}{\partial a_{N \lambda_{s}}} \\
& \times \int_{0}^{\infty} \cdots \int_{0}^{\infty} \frac{d \alpha_{j} \alpha_{j}^{\nu_{j}-1}}{[D(\alpha)]^{d / 2}} \exp \left[i\left(\frac{Q\left(\left\{\overline{s_{i}}\right\}, \alpha\right)}{D(\alpha)}-\sum_{l=1}^{N} \alpha_{l}\left(\bar{m}_{l}^{2}-i \epsilon\right)\right] \mid a_{j}=0,\right.
\end{aligned}
$$

where

$$
\bar{m}_{l}^{2}=m_{l}^{2}+\frac{a_{l}^{2}}{4 \alpha_{l}^{2}}
$$

$\overline{s_{i}}$ are scalar invariants formed from vectors $\overline{q_{i}}$,

$$
\overline{q_{i}}=q_{i}+\sum_{j} \epsilon_{i j} a_{j} \frac{1}{2 \alpha_{j}}
$$

the $q_{i}$ 's are the external momenta incoming at vertex $i$, and $\epsilon$ is the incidence matrix defined as

$$
\epsilon_{i j}=\left\{\begin{array}{l}
+1 \text { if the oriented line } j \text { points away from the vertex } i \\
-1 \text { if the oriented line } j \text { points toward the vertex } i \\
0 \text { if the line } j \text { does not contain the vertex } i
\end{array}\right.
$$

Differentiation with respect to $a_{i}$ will produce external momenta and metric tensors $g_{\mu \nu}$ times some polynomials $R_{s}(\alpha)$ divided by $D(\alpha)$ to some power in the integrand. The polynomials $R_{s}(\alpha)$ have to be converted into operators $R_{s}(\partial)$ and the powers of $D(\alpha)$ are absorbed into the redefinition of $d$. In this way any tensor integral will be expressed as a sum over a set of tensors formed from external vectors and metric tensors multiplied by a combination of scalar integrals with the shifted value of $d$. At the one-loop level such a representation was already proposed in [14].

Tensor integrals in momentum space can be written in terms of scalar ones without direct appeal to the parametric representation (9). The procedure described above may be derived from the formula

$$
\prod_{i=1}^{L} \int d^{d} k_{i} \prod_{j=1}^{N} P_{\bar{k}_{j}}^{\nu_{j}} \prod_{j} \prod_{r=1}^{n_{1}} \bar{k}_{1 \mu_{r}} \ldots \prod_{s=1}^{n_{N}} \bar{k}_{N \lambda_{s}}=T\left(q, \partial, \mathbf{d}^{+}\right) \prod_{i=1}^{L} \int d^{d} k_{i} \prod_{j=1}^{N} P_{\bar{k}_{j}, m_{j}}^{\nu_{j}},
$$

where the tensor operator $T$ is

$$
T\left(q, \partial, \mathbf{d}^{+}\right)=\frac{e^{-i Q\left(\left\{\bar{s}_{i}\right\}, \alpha\right) \rho}}{(-1)^{n_{1}+\cdots+n_{N}}} \prod_{r=1}^{n_{1}} \frac{\partial}{\partial a_{1 \mu_{r}}} \cdots \prod_{s=1}^{n_{N}} \frac{\partial}{\partial a_{N \lambda_{s}}} \exp \left(i\left[Q\left(\left\{\overline{s_{i}}\right\}, \alpha\right)-\sum_{l=1}^{N} \frac{a_{l}^{2}}{4 \alpha_{l}} D(\alpha)\right] \rho\right) \mid \begin{gathered}
a_{j}=0 \\
\alpha_{j}=i \partial_{j} \\
\rho=(-1 / \pi)^{L} \mathbf{d}^{+}
\end{gathered} .
$$


Here $\mathbf{d}^{+}$is the operator shifting the value of the space-time dimension of the integral by two units: $\mathbf{d}^{+} G^{(d)}=G^{(d+2)}$. As before we have to assume that at the beginning all propagators have different masses and after applying the operator $T$ to the integral one should set masses equal to the required ones.

The representation (12) for tensor integrals may give a solution of the problem of irreducible numerators, i.e., the appearance of scalar invariants in the numerator which are absent in the scalar propagators. This kind of integral can be expressed in terms of scalar integrals without numerators but with the changed space-time dimension. Therefore the combined set of recurrence relations, i.e., relations with the changed $d$ and those obtained from integration by parts, should be used to reduce all integrals to the set of scalar master integrals. In [10] we presented a method for the small momentum expansion of multiloop scalar integrals based on $d$ recurrences. This method from the very beginning does not produce integrals with irreducible numerators although those appear in traditional methods for the small momentum expansion. In principle irreducible numerators can be regarded as propagators raised to negative powers and the corresponding integrals can be considered as object in a more complicated class of integrals with additional new denominators. In our approach one remains in the same class of functions, satisfying generalized recurrence relations, i.e., recurrence relations derived from the method of integration by parts plus relations connecting integrals with different $d$.

Having representation (12) at hand, we can now state the procedure for obtaining new, generalized recurrence relations. The starting identity

$$
\prod_{i=1}^{L} \int d^{d} k_{i} \frac{\partial}{\partial k_{r \mu}}\left\{\left(\sum_{l} x_{l} \bar{k}_{l \mu}\right) \prod_{j=1}^{N} P_{\bar{k}_{j}, m_{j}}^{\nu_{j}}\right\} \equiv 0,
$$

where $x_{l}$ are arbitrary constants, written in this form turns out to be rather convenient for the derivation. After performing the differentiation one would usually express scalar products with integration momenta in terms of invariants that occur in the denominator of the propagators. At this point we propose to change the derivation and write all or only some of integrals containing scalar products with loop momenta as a combination of integrals with changed $d$. In this way we produce many relations including integrals with changed exponents of scalar propagators and changed values of the space-time dimension. Combining the different relations [or choosing in a proper way $x_{l}$ in Eq. (14)] one can try to find the most optimal set of relations for the reduction of the concrete class of integrals to the set of basic "master'" integrals. In fact, the method of integration by parts corresponds to some specific representation of scalar products in Eq. (14). Our derivation is more general and it includes an integration by parts method [5] as a particular case.

We expect that solutions of the generalized recurrence relations and those obtained by the method of integration by parts will be of the same complexity. The fact that in case we know the explicit result for an integral in terms of hypergeometric functions, $d / 2$ appears in the same manner as any exponent of the propagators, can be considered as a confirmation of this statement. However, further investigation of this problem is needed.
Several remarks are in order. All relations which connect integrals with shifted $d$ are valid for arbitrary momenta and masses and also for their real and imaginary parts. We want to stress here that in general scalar products in Eq. (8) must be considered as independent variables. One cannot use any restrictions valid for some specific values of $d$.

All relations obtained with the help of the parametric representation may be profitably used in the frame of momentum as well as configuration space.

The minimal change of the space-time parameter is 2 . From concrete examples [7-9], when the result is known in terms of hypergeometric functions, one can observe that changing $d$ by \pm 2 we obtain contiguous functions and therefore may hope to find relations between integrals. The change of $d$ by \pm 1 shifts the parameters of the hypergeometric functions by $\pm 1 / 2$, producing functions which do not belong to the class of contiguous functions. In general there are no relations between those functions.

Application of the differential operators to the integral will increase the powers of the denominators. To simplify the right-hand side (RHS) of Eq. (8) the CT recurrence relations can be used. In general every integral in $d-2$ dimensions can be reduced to combinations of a rational function of scalar products of external momenta, masses, and $d$ and rational multiples of master integrals $I_{j}^{(d)}\left(\left\{s_{i}\right\},\left\{m_{s}^{2}\right\}\right)$ :

$$
G^{(d-2)}\left(\left\{s_{i}\right\},\left\{m_{s}^{2}\right\}\right)=\sum_{j} C_{j}\left(\left\{s_{i}\right\},\left\{m_{s}^{2}\right\}, d\right) I_{j}^{(d)}\left(\left\{s_{i}\right\},\left\{m_{s}^{2}\right\}\right)
$$

The set of basic integrals in $d-2$ dimensions can be expressed in terms of the same integrals in $d$ dimensions:

$$
I_{k}^{(d-2)}\left(\left\{s_{i}\right\},\left\{m_{s}^{2}\right\}\right)=\sum_{j} B_{k j}\left(\left\{s_{i}\right\},\left\{m_{s}^{2}\right\}, d\right) I_{j}^{(d)}\left(\left\{s_{i}\right\},\left\{m_{s}^{2}\right\}\right)
$$

This relation can be inverted and therefore we will get a representation of an arbitrary $d$-dimensional basic scalar integrals in terms of $(d-2)$-dimensional ones. Such a representation may be useful for some practical calculations. The most evident practical application of the relation connecting integrals with different $d$ : Having the $\varepsilon$ expansion for some particular $d_{0}$ we can find similar expansion in $d_{0} \pm 2 l$ ( $l$ integer) dimensions.

The proposed relations can be used, for example, in the evaluation of massless propagator type $(2-2 \varepsilon)$ dimensional integrals using the package MINCER [3] written for $(4-2 \varepsilon)$-dimensional integrals. The polynomials $Q\left(\left\{s_{i}\right\}, \alpha\right), D(\alpha)$ can be easily constructed by means of a computer program for any particular integral [15].

\section{EXAMPLES}

In this section several illustrative examples of the new recurrence relations will be presented. We start with the oneloop propagator-type diagram with massive particles:

$$
I_{\nu_{1} \nu_{2}}^{(d)}\left(q^{2}, m_{1}^{2}, m_{2}^{2}\right)=\int \frac{d^{d} k_{1}}{\left[i \pi^{d / 2}\right]} P_{k_{1}, m_{1}}^{\nu_{1}} P_{k_{1}-q, m_{2}}^{\nu_{2}} .
$$

In this case $D(\alpha)=\alpha_{1}+\alpha_{2}$ and, therefore, 


$$
\begin{aligned}
I_{\nu_{1} \nu_{2}}^{(d-2)}\left(q^{2}, m_{1}^{2}, m_{2}^{2}\right)= & -\left(\partial_{1}+\partial_{2}\right) I_{\nu_{1} \nu_{2}}^{(d)}\left(q^{2}, m_{1}^{2}, m_{2}^{2}\right) \\
= & -\nu_{1} I_{\nu_{1}+1 \nu_{2}}^{(d)}\left(q^{2}, m_{1}^{2}, m_{2}^{2}\right) \\
& -\nu_{2} I_{\nu_{1} \nu_{2}+1}^{(d)}\left(q^{2}, m_{1}^{2}, m_{2}^{2}\right)
\end{aligned}
$$

Here and in the following $\alpha_{i}$ is assigned to the line (or propagator) with mass $m_{i}$. We can get another recurrence relation connecting integrals with different $d$ following the prescription given in Sec. II. From the identity

$$
\int d^{d} k_{1} \frac{\partial}{\partial k_{1 \mu}}\left[\left(k_{1}+q\right)_{\mu} P_{k_{1}, m_{1}}^{\nu_{1}} P_{k_{1}-q, m_{2}}^{\nu_{2}}\right] \equiv 0
$$

we obtain (omitting for the moment arguments of $I_{\nu_{1} \nu_{2}}^{(d)}$ )

$$
\begin{aligned}
\nu_{1} \int & \frac{d^{d} k_{1}}{\left[i \pi^{d / 2}\right]}\left(q k_{1}\right) P_{k_{1}, m_{1}}^{\nu_{1}+1} P_{k_{1}-q, m_{2}}^{\nu_{2}} \\
= & \left(\frac{d}{2}-\nu_{1}\right) I_{\nu_{1} \nu_{2}}^{(d)}-\nu_{2} I_{\nu_{1}-1 \nu_{2}+1}^{(d)}-\nu_{1} m_{1}^{2} I_{\nu_{1}+1 \nu_{2}}^{(d)} \\
& +\nu_{2}\left(q^{2}-m_{1}^{2}\right) I_{\nu_{1} \nu_{2}+1}^{(d)} .
\end{aligned}
$$

The integral with the scalar product $\left(q k_{1}\right)$ can be written as a scalar integral with shifted $d$. According to Eqs. (4) and (11) the function $Q$ with auxiliary vectors $a_{1}, a_{2}$, which are related to the lines with masses $m_{1}, m_{2}$, respectively, is

$$
Q(\{\vec{s}\}, \alpha)=\left(q+\frac{a_{1}}{2 \alpha_{1}}-\frac{a_{2}}{2 \alpha_{2}}\right)^{2} \alpha_{1} \alpha_{2} \text {. }
$$

Formulas (12) and (13) for this case yield the relation

$$
\int \frac{d^{d} k_{1}}{\left[i \pi^{d / 2}\right]}\left(q k_{1}\right) P_{k_{1}, m_{1}}^{\nu_{1}+1} P_{k_{1}-q, m_{2}}^{\nu_{2}}=\nu_{2} q^{2} I_{\nu_{1}+1 \nu_{2}+1}^{(d+2)} \text {. }
$$

Inserting Eq. (22) into Eq. (20) we obtain the desired identity

$$
\begin{gathered}
\nu_{1} \nu_{2} q^{2} I_{\nu_{1}+1 \nu_{2}+1}^{(d+2)}-\left(\frac{d}{2}-\nu_{1}\right) I_{\nu_{1} \nu_{2}}^{(d)}+\nu_{2} I_{\nu_{1}-1 \nu_{2}+1}^{(d)} \\
+\nu_{1} m_{1}^{2} I_{\nu_{1}+1 \nu_{2}}^{(d)}-\nu_{2}\left(q^{2}-m_{1}^{2}\right) I_{\nu_{1} \nu_{2}+1}^{(d)} \equiv 0
\end{gathered}
$$

In addition to the above relations two more relations can be obtained from the traditional method of integration by parts:

$$
\begin{aligned}
& 2 \nu_{2} m_{2}^{2} I_{\nu_{1} \nu_{2}+1}^{(d)}+\nu_{1} I_{\nu_{1}-1 \nu_{2}+1}^{(d)}+\nu_{1}\left(m_{1}^{2}+m_{2}^{2}-q^{2}\right) I_{\nu_{1}+1 \nu_{2}}^{(d)} \\
& -\left(d-2 \nu_{2}-\nu_{1}\right) I_{\nu_{1} \nu_{2}}^{(d)}=0, \\
& \nu_{1} I_{\nu_{1}+1 \nu_{2}-1}^{(d)}-\nu_{2} I_{\nu_{1}-1 \nu_{2}+1}^{(d)}-\nu_{1}\left(m_{1}^{2}-m_{2}^{2}+q^{2}\right) I_{\nu_{1}+1 \nu_{2}}^{(d)} \\
& \quad-\nu_{2}\left(m_{1}^{2}-m_{2}^{2}-q^{2}\right) I_{\nu_{1} \nu_{2}+1}^{(d)}+\left(\nu_{2}-\nu_{1}\right) I_{\nu_{1} \nu_{2}}^{(d)}=0 .
\end{aligned}
$$

For the simplest case, at $m_{1}=m_{2}=0$ and $\nu_{1}=\nu_{2}=1$ from Eqs. (18) and (24) we readily get

$$
I_{11}^{(d-2)}\left(q^{2}, 0,0\right)=-2 I_{21}^{(d)}\left(q^{2}, 0,0\right)=\frac{2(d-3)}{q^{2}} I_{11}^{(d)}\left(q^{2}, 0,0\right) .
$$

This formula can be easily verified from the explicit result for $I_{\nu_{1} \nu_{2}}^{(d)}\left(q^{2}, 0,0\right)$.

At $m_{1}=0, m_{2}=m$, from Eqs. (18) and (23) we get simpler relations connecting integrals $I_{\nu_{1} \nu_{2}}^{(d)}\left(q^{2}, 0, m^{2}\right)$ with different $d$ :

$$
\begin{gathered}
\nu_{1} \nu_{2} q^{2} I_{\nu_{1}+1 \nu_{2}+1}^{(d+2)}\left(q^{2}, 0, m^{2}\right)-\left(\frac{d}{2}-\nu_{1}\right) I_{\nu_{1} \nu_{2}}^{(d)}\left(q^{2}, 0, m^{2}\right) \\
+\nu_{2} I_{\nu_{1}-1 \nu_{2}+1}^{(d)}\left(q^{2}, 0, m^{2}\right)-\nu_{2} q^{2} I_{\nu_{1} \nu_{2}+1}^{(d)}\left(q^{2}, 0, m^{2}\right) \equiv 0, \\
I_{\nu_{1} \nu_{2}}^{(d-2)}\left(q^{2}, 0, m^{2}\right)+\nu_{1} I_{\nu_{1}+1 \nu_{2}}^{(d)}\left(q^{2}, 0, m^{2}\right) \\
+\nu_{2} I_{\nu_{1} \nu_{2}+1}^{(d)}\left(q^{2}, 0, m^{2}\right) \equiv 0 .
\end{gathered}
$$

The integral $I_{\nu_{1} \nu_{2}}^{(d)}\left(q^{2}, 0, m^{2}\right)$ is proportional to the Gauss hypergeometric function [7]:

$$
\begin{aligned}
I_{\nu_{1} \nu_{2}}^{(d)}\left(q^{2}, 0, m^{2}\right)= & (-1)^{\nu_{1}+\nu_{2}} \frac{\Gamma\left(\nu_{1}+\nu_{2}-d / 2\right) \Gamma\left(d / 2-\nu_{1}\right)}{\left(m^{2}\right)^{\nu_{1}+\nu_{2}-d / 2} \Gamma(d / 2) \Gamma\left(\nu_{2}\right)} \\
& \times{ }_{2} F_{1}\left[\begin{array}{c}
\nu_{1}, \nu_{1}+\nu_{2}-\frac{d}{2} ; \\
\frac{d}{2} ;
\end{array}\right] .
\end{aligned}
$$

As is well known there are 15 relations of Gauss between contiguous functions ${ }_{2} F_{1}$. Substituting Eq. (27) into Eq. (24) one can find correspondence between the $\mathrm{CT}$ recurrence relations and only six relations of Gauss. The reason is obvious - in the CT relations the third parameter of ${ }_{2} F_{1}$ in Eq. (27) does not change and therefore all corresponding relations for contiguous functions cannot be reproduced. If we include into consideration also identities (26), we cover all 15 relations, though in principle, to reduce the integrals $I_{\nu_{1} \nu_{2}}^{(d)}\left(q^{2}, 0, m^{2}\right)$, with integer $\nu_{1}$ and $\nu_{2}$ to two boundary integrals, it is enough to know only the CT relations.

Now we consider two-loop bubble integrals with three different masses:

$$
J_{\nu_{1} \nu_{2} \nu_{3}}^{(d)}=\frac{1}{\pi^{d}} \iint d^{d} k_{1} d^{d} k_{2} P_{k_{1}, m_{1}}^{\nu_{1}} P_{k_{1}-k_{2}, m_{2}}^{\nu_{2}} P_{k_{2}, m_{3}}^{\nu_{3}}
$$

The function $D(\alpha)$ for this integral is

$$
D(\alpha)=\alpha_{1} \alpha_{2}+\alpha_{1} \alpha_{3}+\alpha_{2} \alpha_{3},
$$

and hence,

$$
J_{\nu_{1} \nu_{2} \nu_{3}}^{(d-2)}=\left(\partial_{12}^{2}+\partial_{13}^{2}+\partial_{23}^{2}\right) J_{\nu_{1} \nu_{2} \nu_{3}}^{(d)} .
$$

In the above formula and in what follows we use the shorthand notation

$$
\partial_{i_{1} \cdots i_{N}}^{N}=\prod_{j=1}^{N} \frac{\partial}{\partial m_{i_{j}}^{2}} .
$$

Let us take, for simplicity, $\nu_{1}=\nu_{2}=\nu_{3}=1$. In this particular case the relation connecting $J_{111}^{(d)}$ with different $d$ first was 
found in [16]. Exploiting the relation obtained by the method of integration by parts

$$
\begin{aligned}
\Delta \partial_{1} J_{111}^{(d)}= & \frac{1}{2}(d-3)\left(\partial_{1} \Delta\right) J_{111}^{(d)}+(d-2) J_{011}^{(d)} \\
& +\frac{(d-2)}{4 m_{1}^{2}}\left[\left(\partial_{3} \Delta\right) J_{101}^{(d)}+\left(\partial_{2} \Delta\right) J_{110}^{(d)}\right]
\end{aligned}
$$

where

$$
\Delta=m_{1}^{4}+m_{2}^{4}+m_{3}^{4}-2 m_{1}^{2} m_{2}^{2}-2 m_{1}^{2} m_{3}^{2}-2 m_{2}^{2} m_{3}^{2},
$$

and similar relations for $\partial_{2} J_{111}^{(d)}, \partial_{3} J_{111}^{(d)}$, from Eq. (30) we reproduce the relationship between integrals with different $d$ given in [16]:

$$
\begin{aligned}
\Delta J_{111}^{(d-2)}= & -(d-2)(d-3) J_{111}^{(d)}+\frac{1}{2} \Gamma^{2}\left(2-\frac{d}{2}\right)\left[s_{1} s_{2} \partial_{3} \Delta\right. \\
& \left.+s_{1} s_{3} \partial_{2} \Delta+s_{2} s_{3} \partial_{1} \Delta\right]
\end{aligned}
$$

where $s_{i}=m_{i}^{d-4}$.

One can easily obtain another recurrence relation for the integral (28) with different $d$. From the identity

$$
\begin{array}{r}
\iint d^{d} k_{1} d^{d} k_{2}\left(\frac{\partial}{\partial k_{1 \mu}}+\frac{\partial}{\partial k_{2 \mu}}\right)\left[k_{1 \mu} P_{k_{1}, m_{1}}^{\nu_{1}} P_{k_{1}-k_{2}, m_{2}}^{\nu_{2}} P_{k_{2}, m_{3}}^{\nu_{3}}\right] \\
\equiv 0,
\end{array}
$$

keeping the scalar product $\left(k_{1} k_{2}\right)$ untouched, we get

$$
\begin{gathered}
\frac{\nu_{3}}{\pi^{d}} \iint d^{d} k_{1} d^{d} k_{2}\left(k_{1} k_{2}\right) P_{k_{1}, m_{1}}^{\nu_{1}} P_{k_{1}-k_{2}, m_{2}}^{\nu_{2}} P_{k_{2}, m_{3}}^{\nu_{3}+1} \\
+\nu_{1} m_{1}^{2} J_{\nu_{1}+1 \nu_{2} \nu_{3}}^{(d)}-\left(\frac{d}{2}-\nu_{1}\right) J_{\nu_{1} \nu_{2} \nu_{3}}^{(d)} \equiv 0
\end{gathered}
$$

The first integral in Eq. (35) can be expressed in terms of integrals with another value of the space-time dimension $d$ by using formula (13) with

$$
Q(\{s\}, \alpha)=\frac{1}{4}\left(\frac{a_{1}}{\alpha_{1}}-\frac{a_{2}}{\alpha_{2}}-\frac{a_{3}}{\alpha_{3}}\right)^{2} \alpha_{1} \alpha_{2} \alpha_{3} .
$$

Here again vectors $a_{i}$ correspond to lines with mass $m_{i}$. In order to obtain the integral with the scalar product $\left(k_{1} k_{2}\right)$ in the integrand one has to differentiate with respect to $a_{1}$ and $a_{3}$ which leads to

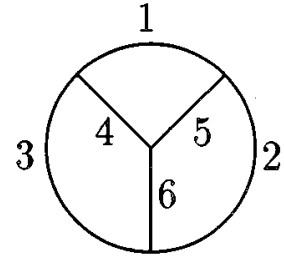

FIG. 2. Three-loop bubble diagram.

$$
\begin{aligned}
& \frac{1}{\pi^{d}} \int d^{d} k_{1} d^{d} k_{2}\left(k_{1} k_{2}\right) P_{k_{1}, m_{1}}^{\nu_{1}} P_{k_{1}-k_{2}, m_{2}}^{\nu_{2}} P_{k_{2}, m_{3}}^{\nu_{3}+1} \\
& \quad=\frac{1}{2} \nu_{2} d J_{\nu_{1} \nu_{2}+1 \nu_{3}+1}^{(d+2)} .
\end{aligned}
$$

Substituting Eq. (37) into Eq. (35) we obtain

$$
\nu_{2} \nu_{3} d J_{\nu_{1} \nu_{2}+1 \nu_{3}+1}^{(d+2)}+2 \nu_{1} m_{1}^{2} J_{\nu_{1}+1 \nu_{2} \nu_{3}}^{(d)}-\left(d-2 \nu_{1}\right) J_{\nu_{1} \nu_{2} \nu_{3}}^{(d)} \equiv 0
$$

This identity was used to evaluate the coefficients in the small momentum expansion of the two-loop master diagram with all masses equal by the method proposed in [10].

Now we turn to the three-loop case and consider for simplicity bubble diagrams. At the three-loop level, bubble diagrams in general have the topology shown in Fig. 2, where each line corresponds to a scalar propagator with an arbitrary exponent.

The relation between $d$ - and $(d-2)$-dimensional threeloop vacuum integrals with all masses arbitrary is

$$
\begin{aligned}
G^{(d-2)}\left(\left\{m_{s}^{2}\right\}\right)= & -\frac{1}{\pi^{3}}\left(\partial_{123}^{3}+\partial_{124}^{3}+\partial_{126}^{3}+\partial_{135}^{3}+\partial_{136}^{3}+\partial_{145}^{3}\right. \\
& +\partial_{146}^{3}+\partial_{156}^{3}+\partial_{234}^{3}+\partial_{235}^{3}+\partial_{245}^{3}+\partial_{246}^{3}+\partial_{256}^{3} \\
& \left.+\partial_{345}^{3}+\partial_{346}^{3}+\partial_{356}^{3}\right) G^{(d)}\left(\left\{m_{s}^{2}\right\}\right)
\end{aligned}
$$

The numbering of the masses corresponds to the numbering of the lines in Fig. 2.

Three-loop master bubble integrals encountered in the small momentum expansion of the QED photon propagator were studied in [17]. Every integral in this case can be expressed in terms of three basic structures. The only nontrivial combination of two integrals taken as one of such structure was

$$
\begin{aligned}
m^{3 d-12} B_{4}^{(d)}= & -\frac{1}{4}(d-2)(d-3) \iiint \frac{d^{d} k_{1} d^{d} k_{2} d^{d} k_{3}}{\left[i \pi^{d / 2} \Gamma(3-d / 2)\right]^{3}} P_{k_{2}, m} P_{k_{3}, m} P_{k_{2}-k_{3}, 0} \\
& \times\left[P_{k_{1}, m} P_{k_{1}-k_{3}, 0} P_{k_{1}-k_{2}, 0}-P_{k_{1}, 0} P_{k_{1}-k_{3}, m} P_{k_{1}-k_{2}, m}\right] .
\end{aligned}
$$

By using Eq. (39) and the method of integration by parts we find the following relation for $B_{4}^{(d)}$ :

$$
\begin{aligned}
B_{4}^{(d-2)}= & \frac{3(d-4)^{2}(d-5)(3 d-14)(3 d-16)}{16(d-6)^{5}} B_{4}^{(d)}+\frac{33 d^{3}-367 d^{2}+1170 d-864}{8(d-4)^{2}(d-6)^{5}} \\
& -\frac{7\left(8 d^{2}-80 d+195\right)}{(d-4)^{2}(d-6)^{5}} \frac{\Gamma(d / 2-1) \Gamma^{2}(6-d) \Gamma(9-3 d / 2)}{\Gamma^{2}(3-d / 2) \Gamma(12-2 d)} .
\end{aligned}
$$


We observe that the relation includes $B_{4}^{(d)}$ itself and two additional terms with a trivial $d$ dependence. The results for other diagrams of the three-loop photon propagator look similar; i.e., they exhibit three terms of a structure like in Eq. (41).

Other useful three-loop vacuum integrals were introduced in [18]:

$$
\begin{gathered}
m^{3 d-12} D_{3}=\iiint \frac{d^{d} k_{1} d^{d} k_{2} d^{d} k_{3}}{\left[i \pi^{d / 2} \Gamma(3-d / 2)\right]^{3}} P_{k_{1}, 0} P_{k_{2}, 0} P_{k_{3}, m} P_{k_{1}-k_{3}, 0} P_{k_{1}-k_{2}, m} P_{k_{2}-k_{3}, m}, \\
m^{3 d-12} B_{5}=\iiint \frac{d^{d} k_{1} d^{d} k_{2} d^{d} k_{3}}{\left[i \pi^{d / 2} \Gamma(3-d / 2)\right]^{3}} P_{k_{1}, m} P_{k_{3}, m} P_{k_{1}-k_{2}, m} P_{k_{2}-k_{3}, 0} .
\end{gathered}
$$

These integrals occurred in the evaluation of the three-loop QCD correction to the electroweak $\rho$ parameter. The space-time recurrence relation for $D_{3}^{(d)}$ looks more complicated than that for $B_{4}^{(d)}$ and it includes also $B_{5}^{(d)}$ :

$$
\begin{aligned}
D_{3}^{(d-2)}= & -\frac{16\left(63 d^{3}-832 d^{2}+3622 d-5176\right)}{(d-4)^{4}(d-5)^{2}(d-6)^{3}}-\frac{64 \Gamma(d / 2-1) \Gamma(5-d)}{(d-4)^{3}(d-5)(d-6)^{3} \Gamma(3-d / 2)}\left[1+\frac{(7 d-32) \Gamma(d / 2-1) \Gamma(7-3 d / 2)}{3 \Gamma^{2}(3-d / 2)}\right. \\
& \left.+\frac{\left(37 d^{2}-350 d+828\right) \Gamma(5-d) \Gamma(7-3 d / 2)}{24(2 d-9) \Gamma(3-d / 2) \Gamma(9-2 d)}\right]-\frac{4(d-2)(d-3)(3 d-8)(3 d-10)}{9(d-4)(d-6)^{3}} B_{5}^{(d)} \\
& -\frac{4(d-2)(d-3)(d-4)}{(d-6)^{3}} D_{3}^{(d)} .
\end{aligned}
$$

The corresponding relation for $B_{5}^{(d)}$ is simpler and reads

$$
B_{5}^{(d-2)}=-\frac{4(d-2)(d-3)(3 d-8)(3 d-10)}{9(d-4)(d-6)^{3}} B_{5}^{(d)}-\frac{64(15 d-52)}{9(d-4)^{4}(d-6)^{3}} .
$$

It can be obtained from Eq. (39) by observing that $\partial_{2} G^{(d)}=\partial_{4} G^{(d)}=0$, which holds because $B_{5}^{(d)}$ corresponds to the diagram Fig. 2 with contracted second and fourth lines. For $B_{5}^{(d)}$ we found an explicit expression in terms of hypergeometric functions ${ }_{3} F_{2}$ and ${ }_{2} F_{1}$ with the argument $1 / 4$, satisfying relation (45).

Relations (41), (44), and (45) can be used for the computation of the coefficients in the small momentum expansion of Feynman diagrams by the method proposed in [10].

A more detailed analysis of the generalized recurrence relations for two- and three-loop diagrams will be given in a future publication. Several examples of these recurrence relations one can find also in [10].

\section{RELATIONS FOR $\boldsymbol{n}$-POINT ONE-LOOP INTEGRALS}

In this section we consider scalar one-loop integrals depending on $n-1$ external momenta:

$$
I_{n}^{(d)}=\int \frac{d^{d} q}{\pi^{d / 2}} \prod_{j=1}^{n} \frac{1}{[j]^{v_{j}}},
$$

where

$$
[j]=\left(q-p_{j}\right)^{2}-m_{j}^{2}+i \epsilon \quad \text { for } j<n \text { and }[n]=q^{2}-m_{n}^{2}+i \epsilon .
$$

The corresponding diagram and the convention for the momenta are given in Fig. 3.
For the diagram in Fig. 3 the function $D(\alpha)$ is a polynomial linear in $\alpha_{j}$,

$$
D(\alpha)=\sum_{j=1}^{n} \alpha_{j}
$$

and, hence,

$$
I_{n}^{(d-2)}=-\sum_{j=1}^{n} \partial_{j} I_{n}^{(d)}
$$

To get rid of the derivatives in this relation we use the method of integration by parts. Let us consider the identity

$$
\int d^{d} q \frac{\partial}{\partial q_{\mu}}\left[\frac{x_{n} q_{\mu}+\sum_{i=1}^{n-1} x_{i} p_{i \mu}}{\Pi_{j=1}^{n}[j]^{\nu_{j}}}\right] \equiv 0,
$$

which is valid for arbitrary $x_{i}$. Upon differentiation and expressing scalar products in the numerator in terms of invariants $[j]$ in the denominator, we get

$$
\begin{aligned}
& \int d^{d} q \frac{1}{\prod_{i=1}^{n}[i]^{\nu_{i}}}\left\{x_{n}\left(d-\nu_{n}-\sum_{j=1}^{n} \nu_{j}\right)\right. \\
& +\sum_{j=1}^{n-1} x_{j}\left(\nu_{j}-\nu_{n}+[j] \sum_{k=1, k \neq j}^{n-1} \frac{\nu_{k}}{[k]}\right) \\
& \left.-[n] \sum_{j=1}^{n-1} \frac{\nu_{j}}{[j]} \sum_{i=1}^{n} x_{i}+\sum_{j=1}^{n} \frac{R_{j}^{(n)}\left(\left\{x_{i}\right\}\right)}{[j]}\right\} \equiv 0,
\end{aligned}
$$


where $R_{j}^{(n)}\left(\left\{x_{i}\right\}\right)=\sum_{i=1}^{n} R_{i j}^{(n)} x_{i}$ with

$$
\begin{gathered}
R_{n i}^{(n)}=-m_{i}^{2}-m_{n}^{2}+p_{i}^{2}, \quad R_{i n}^{(n)}=m_{i}^{2}-m_{n}^{2}-p_{i}^{2} \quad \text { for } i<n, \\
R_{i j}^{(n)}=m_{i}^{2}-m_{n}^{2}-p_{i}^{2}+2 p_{i} p_{j}, \quad \text { for } i, j<n \\
R_{n n}^{(n)}=-2 m_{n}^{2} .
\end{gathered}
$$

The integral related to the last sum in (51) can be made proportional to the RHS of Eq. (49). This requires to find $x_{i}$ such that

$$
R_{j}^{(n)}\left(\left\{x_{i}\right\}\right)=1, \quad j=1, \ldots, n .
$$

Imposing these conditions we obtain a system of $n$ equations for $x_{i}$. The solution of the system reads

$$
x_{i}=\frac{1}{2} \partial_{i} \ln \Delta_{n} \text { for } i<n, x_{n}=\frac{G_{n-1}}{2 \Delta_{n}},
$$

where $G_{n}$ is the Gram determinant,

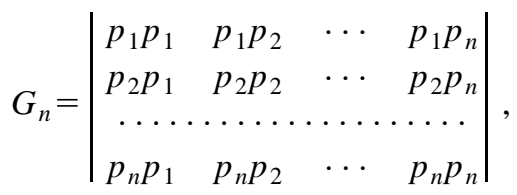

and $\Delta_{n}$ is proportional to the determinant,

$$
\Delta_{n}=-\frac{1}{2^{n}} \operatorname{det}(C)
$$

of the $n \times n$ matrix $C$,

$$
C_{i j}=m_{i-1}^{2}+m_{j-1}^{2}-\left(p_{i-1}-p_{j-1}\right)^{2},
$$

where it is assumed that $m_{0}=m_{n}$ and $p_{0}=0$. Substituting $x_{i}$ into Eq. (51), we obtain the following relation between $d$-dimensional and $(d-2)$-dimensional integrals:

$$
\begin{aligned}
& {\left[\sum_{j=1}^{n-1}\left(\nu_{j}-\nu_{n}\right)\left(\partial_{j} \Delta_{n}\right)+\left(d-\nu_{n}-\sum_{j=1}^{n} \nu_{j}\right) G_{n-1}\right] I_{n}^{(d)}} \\
& \quad=\sum_{j=1}^{n}\left(\partial_{j} \Delta_{n}\right) I_{n, j}^{(d-2)}+2 \Delta_{n} I_{n}^{(d-2)} .
\end{aligned}
$$

The index $j$ of $I_{n, j}^{(d-2)}$ means that the factor $1 /[j]^{\nu_{j}}$ in the integrand must be changed into $[j] /[j]^{\nu_{j}}$. For $\nu_{j}=1$ we obtain the simpler relation

$$
(d-n-1) G_{n-1} I_{n}^{(d)}=\sum_{j=1}^{n}\left(\partial_{j} \Delta_{n}\right) I_{n, j}^{(d-2)}+2 \Delta_{n} I_{n}^{(d-2)} .
$$

For $d=6, n \geqslant 6$ and assuming that external momenta are four dimensional the Gram determinant $G_{n-1}$ vanishes and hence the term with $I_{n}^{(6)}$ drops out and we get a relationship between four-dimensional integrals with $n$ legs and integrals with $n-1$ legs. Such a relation was first obtained in [19] (see also [20]). When $d=6$ and $n=5$ the left-hand side (LHS) of Eq. (58) is zero and we arrive at a formula for reducing

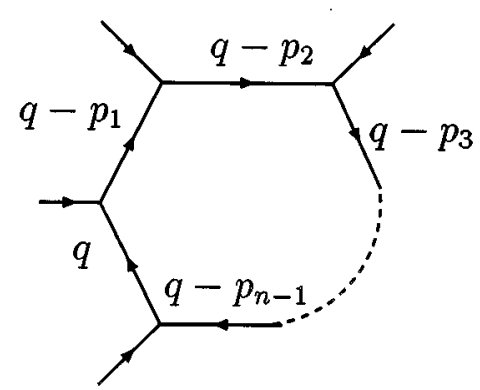

FIG. 3. One-loop diagram with $n$-legs.

four-dimensional pentagon integrals to box integrals. This relation was first derived in [21]. For arbitrary $d$ and $n$, Eq. (58) was obtained by a different method in [22]. Equation (57) as far as we know is new.

It is evident that by using Eq. (8) and integration by parts one can also derive relations similar to Eq. (58) for multiloop integrals.

Equation (57) can be used in the reduction of the one-loop tensor integrals to scalar ones. In [14] an explicit general formula for one-loop tensor integrals was derived:

$$
\begin{aligned}
\int d^{d} q \frac{q_{\mu_{1}} \cdots q_{\mu_{M}}}{\Pi_{j=1}^{n}[j]^{\nu_{j}}}= & \sum_{\lambda, k_{1}, \ldots, k_{n-1}} \frac{(-1)^{M-\lambda}}{2^{\lambda}} \\
& 2 \lambda+\sum k_{i}=M \\
& \times\left\{[g]^{\lambda}\left[p_{1}\right]^{k_{1}} \cdots\left[p_{n-1}\right]^{k_{n-1}}\right\}_{\mu_{1} \cdots \mu_{M}} \\
& \times \int \frac{d^{d+2 M-2 \lambda} q}{\pi^{M-\lambda}[n]^{\nu_{n}}} \prod_{j=1}^{n} \frac{\left(\nu_{j}\right)_{k_{j}}}{[j]^{\nu_{j}+k_{j}}},
\end{aligned}
$$

where $(\nu)_{k} \equiv \Gamma(\nu+k) / \Gamma(\nu)$ is the Pochhammer symbol. The shorthand notation $\left\{[g]^{\lambda}\left[p_{1}\right]^{k_{1}} \cdots\left[p_{n-1}\right]^{k_{n-1}}\right\}_{\mu_{1}} \cdots \mu_{M}$ corresponds to the symmetrical (with respect to $\mu_{1} \cdots \mu_{M}$ ) tensor combination, each term of which is constructed from $\lambda$ metric tensors $g, k_{1}$ momenta $p_{1}, \ldots, k_{n-1}$ momenta $p_{n-1}$. For example,

$$
\left\{g p_{1}\right\}_{\mu_{1} \mu_{1} \mu_{3}}=g_{\mu_{1} \mu_{2}} p_{1 \mu_{3}}+g_{\mu_{1} \mu_{3}} p_{1 \mu_{2}}+g_{\mu_{2} \mu_{3}} p_{1 \mu_{1}} .
$$

In Eq. (59), $\lambda, k_{i} \geqslant 0, \max k_{i}=M, \max \lambda=[M / 2]$ (integer part of $M / 2$ ). For more details see [14]. Thus, the procedure of calculating one-loop diagrams will be as follows. One should use first Eq. (59), perform contractions of indices, and then using (57) reduce all scalar integrals with the changed dimensions to the $d=4-2 \varepsilon$ dimensional set of integrals. After that scalar integrals should be reduced to the set of master integrals by making use of recurrence relations obtained by the method of integration by parts.

\section{EVALUATION OF ONE-LOOP INTEGRALS USING SPACE-TIME RECURRENCE RELATIONS}

With the help of Eqs. (15) and (16) one can obtain relations that include only one particular integral with different shifts in $d$ :

$$
\sum_{k} B_{k}\left(\left\{s_{i}\right\},\left\{m_{s}^{2}\right\}, d\right) I^{(d-k)}\left(\left\{s_{i}\right\},\left\{m_{s}^{2}\right\}\right)=I_{0}\left(\left\{s_{i}\right\},\left\{m_{s}^{2}\right\}, d\right),
$$



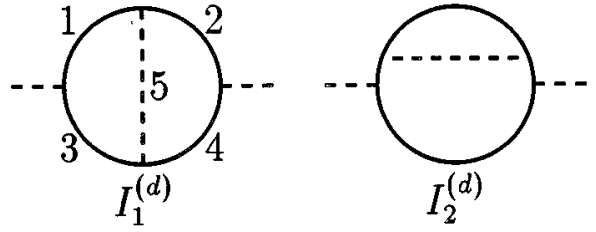

FIG. 4. Two-loop scalar diagrams with massive loops.

where $I_{0}\left(\left\{s_{i}\right\},\left\{m_{s}^{2}\right\}, d\right)$ is some explicitly known expression. It can be eliminated from the equation giving rise to higherorder recurrence relations. For an explicit solution Eq. (60) looks simpler than the ones obtained by the method of integration by parts. In this section we will demonstrate how the space-time recurrence relations proposed in the present paper can be used for evaluating integrals.

We consider the one-loop propagator-type integral

$$
I^{(d)}=m^{4-d} \int \frac{d^{d} k_{1}}{\left[i \pi^{d / 2}\right]} P_{k_{1}, m} P_{k_{1}-q, m} .
$$

By using Eq. (8) and the first relation of Eq. (24) we obtain

$$
I^{(d-2)}=\frac{2(d-3) m^{2}}{\left(q^{2}-4 m^{2}\right)} I^{(d)}-\frac{2 \Gamma(2-d / 2) m^{2}}{\left(q^{2}-4 m^{2}\right)} .
$$

Introducing

$$
\bar{I}^{(d)}=i^{-d}\left(\frac{m^{2}}{4 m^{2}-q^{2}}\right)^{d / 2} \frac{\Gamma(d-2)}{\Gamma(d / 2-1)} I^{d},
$$

we get the simpler equation

$$
\bar{I}^{(d-2)}=\bar{I}^{(d)}-\left(\frac{m^{2}}{4 m^{2}-q^{2}}\right)^{d / 2} \frac{\Gamma(d-3) \Gamma(2-d / 2)}{i^{d} \Gamma(d / 2-1)} .
$$

Without loss of generality, one can parametrize $d$ as $d=2 l-2 \varepsilon$, where $l$ is an integer number and $\varepsilon$ is an arbitrary small number. The inhomogeneous term in Eq. (64) can be absorbed by a redefinition of $\bar{I}^{(d)}$ :

$$
\begin{aligned}
\bar{I}^{(2 l-2 \varepsilon)}= & \sum_{j=0}^{l}\left(\frac{m^{2}}{4 m^{2}-q^{2}}\right)^{j-\varepsilon} \frac{\Gamma(2 j-2 \varepsilon-3) \Gamma(2-j+\varepsilon)}{i^{2 j-2 \varepsilon} \Gamma(j-1-\varepsilon)} \\
& +C_{\varepsilon}^{l},
\end{aligned}
$$

yielding the following very simple equation for $C_{\varepsilon}^{l}$

$$
C_{\varepsilon}^{l}=C_{\varepsilon}^{l-1} .
$$

Since $l$ in our case is an arbitrary integer we can conclude that $C_{\varepsilon}^{l}$ does not depend on $l$ at all. It can be found, for example, by taking the limit $l \rightarrow \infty$ or $|d| \rightarrow \infty$. Taking the limit $|d| \rightarrow \infty$ is quite a delicate matter. The larger $|d|$, the more divergent becomes the integral. In order to keep the regularization of the integral, we have to consider $\varepsilon$ as complex and $l$ large. The asymptotic value of the integral as $l \rightarrow \infty$ can be found by the method of steepest descent. It depends on the sign of $q^{2}$. From the parametric representation of the integral $I^{(d)}$ one gets, for $q^{2}<0$ and $l \rightarrow \infty$,

$$
\begin{aligned}
I^{(d)} \rightarrow & \Gamma(2-l+\varepsilon)\left(\frac{-\pi m^{2}}{q^{2} l}\right)^{1 / 2}\left(1-\frac{q^{2}}{4 m^{2}}\right)^{l-\varepsilon-3 / 2} \\
& \times[1+O(1 / l)] .
\end{aligned}
$$

At large $l$ and $q^{2}<0$, the sum in Eq. (65) is convergent which allows us to find $C_{\varepsilon}^{l}$ :

$$
\begin{aligned}
C_{\varepsilon}^{l}= & \frac{i^{2 \varepsilon} \pi}{\sin \pi \varepsilon} \frac{m^{4}}{\sqrt{-q^{2}}\left(4 m^{2}-q^{2}\right)^{3 / 2}} \\
& -\sum_{j=0}^{\infty}\left(\frac{m^{2}}{4 m^{2}-q^{2}}\right)^{j-\varepsilon} \frac{\Gamma(2 j-2 \varepsilon-3) \Gamma(2-j+\varepsilon)}{i^{2 j-2 \varepsilon} \Gamma(j-\varepsilon-1)} .
\end{aligned}
$$

Substitution of $C_{\varepsilon}^{l}$ in the above formulas yields

$$
\begin{aligned}
I^{(d)}= & \frac{\sqrt{\pi} \Gamma(d / 2-1) \Gamma(2-d / 2)}{\Gamma((d-1) / 2)} \frac{m}{\sqrt{-q^{2}}}\left(1-\frac{q^{2}}{4 m^{2}}\right)^{(d-3) / 2} \\
& +\Gamma\left(1-\frac{d}{2}\right) \frac{2 m^{2}}{\left(4 m^{2}-q^{2}\right)^{2}} F_{1}\left[\begin{array}{c}
1, \frac{d-1}{2} ; \\
\frac{d}{2} ;
\end{array} \frac{4 m^{2}}{4 m^{2}-q^{2}}\right] .
\end{aligned}
$$

By using the formula for the analytic continuation of the hypergeometric series we get the known result

$$
I^{(d)}=\Gamma\left(2-\frac{d}{2}\right){ }_{2} F_{1}\left[\begin{array}{c}
1,2-\frac{d}{2} ; \frac{q^{2}}{4 m^{2}} \\
\frac{3}{2} ;
\end{array}\right] .
$$

The example considered illustrates the main ideas of how to use the relation (8) for the evaluation of Feynman integrals. The same method applies without modification to more complicated cases, for example, to the one-loop integral (17) with different masses at $\nu_{1}=\nu_{2}=1$ or to the two-loop integral (28) with $\nu_{1}=\nu_{2}=\nu_{3}=1$. The recurrence relations in these cases are very similar to Eq. (62). We applied this technique also to some two- and three-loop integrals. The main difficulty encountered in these computations was to find the asymptotic value for the integral for large $d$. An expansion at large $d$ is frequently used in solid state physics and statistical physics. Our experience shows that it can also be used for the approximate evaluation of individual Feynman integrals. Details of these calculations will be given in a future publication.

\section{THE TWO-LOOP SELF-ENERGY FOR THE $\phi^{3}$ MODEL IN 6-2 $\varepsilon$ DIMENSIONS}

The aim of this section is to show how $d$-recurrence relations can be used to obtain the $\varepsilon$ expansion of the nontrivial integrals in one dimension from the known expansion in another dimension.

We consider two-loop self-energy diagrams which are encountered, for example, in the $\phi^{3}$ model with two massive and one massless scalar fields [23]. At the two-loop level there are two nontrivial diagrams, given in Fig. 4, contributing to the self-energy of the massless field.

The solid lines in Fig. 4 are related to massive particles and the dashed lines to massless ones. The corresponding integrals read 


$$
\begin{gathered}
I_{1}^{(d)}=\iint \frac{d^{d} k_{1} d^{d} k_{2}}{\left[i \pi^{d / 2} \Gamma(4-d / 2)\right]^{2}} \\
\times P_{k_{1}, m} P_{k_{2}, m} P_{k_{1}-q, m} P_{k_{2}-q, m} P_{k_{1}-k_{2}, 0}, \\
I_{2}^{(d)}=\iint \frac{d^{d} k_{1} d^{d} k_{2}}{\left[i \pi^{d / 2} \Gamma(4-d / 2)\right]^{2}} P_{k_{1}, m}^{2} P_{k_{2}, m} P_{k_{2}-q, m} P_{k_{1}-k_{2}, 0} .
\end{gathered}
$$

Using the $\varepsilon$ expansion in $d=4-2 \varepsilon$ dimensions we will find the $\varepsilon$ expansion of these integrals up to $O(\varepsilon)$ at $d=6-2 \varepsilon$. This can be done in three steps.

The first step consists of expressing the integrals $I_{1}^{(d)}$ and $I_{2}^{(d)}$ in terms of basic ones, chosen in [8] to obtain the $d \rightarrow 4$ limit for the two-loop photon propagator. In fact two integrals from this basis are the integral $I_{1}^{(d)}$ itself and its derivative with respect to the mass. The remaining basic integrals were the derivative with respect to the mass of the one-loop scalar integral with two massive lines, its square, and a one-loop vacuum integral with a mass.

Thus, at this stage we need to compute only the integral $I_{2}$. By using the recurrence relations given in [8] for integrals contributing to the two-loop photon propagator, we get

$$
\begin{aligned}
I_{2}^{(d)}= & -\frac{(d-4)}{(d-3)} I_{1}^{(d)}+\frac{m^{2}}{(d-3)}\left(1-\frac{q^{2}}{4 m^{2}}\right)\left(I_{1}^{(d)}\right)^{\prime} \\
& +\frac{m^{2 d-10}}{(d-3)(d-4)} H_{1}^{(d)},
\end{aligned}
$$

where the prime denotes differentiation with respect to $\mathrm{m}^{2}$ and $H_{1}^{(d)}$ is a basic integral occurring in one-loop calculations:

$$
\begin{aligned}
H_{1}^{(d)} & ={ }_{2} F_{1}\left[\begin{array}{c}
1,3-\frac{d}{2} ; \\
\frac{3}{2} ; \\
4 m^{2}
\end{array}\right] \\
& =\frac{1}{d-5}+\frac{(d-6)}{(d-5)}\left(1-\frac{q^{2}}{4 m^{2}}\right) H_{1}^{(d-2)} .
\end{aligned}
$$

The next step is to find relations between the basic integrals $I_{1}^{(d)}$ and $\left(I_{1}^{(d)}\right)^{\prime}$ in $d$ and $d-2$ dimensions. Using Eq. (8), with $D(\alpha)$ given by Eq. (5) we get a system of equations in the form of Eq. (16). This system enables us to find the required relations

$$
\begin{aligned}
& -3 z(d-3)(d-4)^{2}(3 d-14)(3 d-16) I_{1}^{(d)}=\frac{m^{4}}{4}(d-8)^{2}(d-6)\left[-(d-6)(d-4) z^{3}+12(d-4)(2 d-11) z^{2}+12(d-7)\right. \\
& \times(3 d-14) z-16(d-7)(2 d-9)] I_{1}^{(d-2)}+\frac{m^{6}}{4}(d-8)^{2}(d-6)\left[2(d-4) z^{3}\right. \\
& \left.+2(3 d-16) z^{2}-8(9 d-41) z+32(2 d-9)\right]\left(I_{1}^{(d-2)}\right)^{\prime}-\frac{2 m^{2 d-10}}{(d-6)^{2}(4-z)} \\
& \times\left[(d-4)\left(23 d^{2}-226 d+552\right) z^{3}-8(d-4)\left(19 d^{2}-188 d+462\right) z^{2}\right. \\
& \left.+16\left(22 d^{3}-310 d^{2}+1447 d-2238\right) z+64(2 d-9)(d-5)^{2}\right]\left(H_{1}^{(d)}\right)^{2} \\
& +\frac{16 m^{2 d-10}}{(d-6)^{2}(4-z)}\left[\left(19 d^{2}-182 d+432\right) z^{2}-\left(56 d^{2}-552 d+1344\right) z+16\right. \\
& \times(2 d-9)(2 d-11)] H_{1}^{(d)}+\frac{16 m^{2 d-10}}{(d-6)^{2}(d-4)(d-5)(4-z)}\left[(d-6)(d-4)^{2} z^{2}\right. \\
& \left.-2\left(d^{3}+3 d^{2}-98 d+288\right) z-8(d-5)(5 d-28)(2 d-9)\right] \\
& -3 z(d-3)(d-4)^{2}(3 d-14)(3 d-16)\left(I_{1}^{(d)}\right)^{\prime}=\frac{3 m^{2}}{2}(d-4)(d-6)(d-8)^{2}\left[(d-4)(d-5) z^{2}+(7 d-34)(d-7) z-4(2 d-9)\right. \\
& \times(d-7)] I_{1}^{(d-2)}+\frac{3 m^{4}}{4}(d-4)(d-6)(d-8)^{2}\left[(5 d-24) z^{2}-4(7 d-33) z\right. \\
& +16(2 d-9)]\left(I_{1}^{(d-2)}\right)^{\prime}+\frac{24(d-4) m^{2 d-12}}{(4-z)(d-6)^{2}}\left[(d-4)\left(7 d^{2}-70 d+174\right) z^{2}\right. \\
& \left.-\left(30 d^{3}-424 d^{2}+1986 d-3084\right) z-8(2 d-9)(d-5)^{2}\right]\left(H_{1}^{(d)}\right)^{2} \\
& -\frac{96(d-4) m^{2 d-12}}{(4-z)(d-6)^{2}}[(d-4)(d-6) z-4(2 d-9)(2 d-11)] H_{1}^{(d)} \\
& +\frac{48(7 d-32)(d-6) z-192(2 d-9)(5 d-28)}{m^{12-2 d}(4-z)(d-6)^{2}},
\end{aligned}
$$


where $z=q^{2} / m^{2}$.

The final step is to perform the $\varepsilon$ expansion up to a constant term. It is remarkable that the most complicated integrals $I_{1}^{(d-2)}$ and $\left(I_{1}^{(d-2)}\right)^{\prime}$ at $d=6-2 \varepsilon$ give contribution starting from the first order in $\varepsilon$ and therefore may be disregarded in the considered approximation. To calculate $I_{1}^{(d)}$ and $I_{2}^{(d)}$ up to a constant term in $\varepsilon$ we need to expand $H_{1}^{(d)}$ to second order. The required expansion is

$$
H_{1}^{(6-2 \varepsilon)}=1+\varepsilon\left[2+\frac{1}{a} \ln (v)\right]+\varepsilon^{2}\left[4+\frac{\pi^{2}}{6 a}+\frac{1}{2 a} \ln ^{2}(v)+\frac{2}{a} \ln (v)-\frac{1}{a} \ln ^{2}\left(\frac{1+a}{2}\right)-\frac{2}{a} \operatorname{Li}_{2}\left(\frac{1+a}{2}\right)\right],
$$

where

$$
a^{2}=\frac{q^{2}}{q^{2}-4 m^{2}}, \quad v=\frac{1-a}{1+a}, \quad \text { and } \operatorname{Li}_{2}(x)=-\int_{0}^{x} \frac{\ln (1-y) d y}{y},
$$

from which we obtain

$$
\begin{aligned}
m^{4 \varepsilon-2} I_{1}^{(6-2 \varepsilon)}= & \frac{3-a^{2}}{6 \varepsilon^{2}\left(1-a^{2}\right)}+\frac{31-7 a^{2}}{12 \varepsilon\left(1-a^{2}\right)}+\frac{19}{24}-\frac{1}{12 a^{2}}+\frac{47}{6\left(1-a^{2}\right)}-\frac{2 h(a)}{3 a\left(1-a^{2}\right)} \\
& -\left(\frac{5}{48}-\frac{1}{3 a}-\frac{7}{24 a^{2}}+\frac{1}{48 a^{4}}-\frac{1}{3(1-a)}\right) \ln ^{2}(v)+\frac{1}{12}\left(\frac{43}{a}-\frac{1}{a^{3}}+\frac{48 a}{\left(1-a^{2}\right)}\right) \ln (v), \\
m^{4 \varepsilon-2} I_{2}^{(6-2 \varepsilon)}= & -\frac{a^{2}}{18 \varepsilon^{2}\left(1-a^{2}\right)}-\frac{15+28 a^{2}}{108 \varepsilon\left(1-a^{2}\right)}+\frac{389}{324}-\frac{1}{36 a^{2}}-\frac{1207}{648\left(1-a^{2}\right)}-\frac{1-3 a^{2}}{18 a\left(1-a^{2}\right)} h(a) \\
& +\frac{1}{144}\left(10+\frac{4}{a}-\frac{1}{a^{2}}-\frac{1}{a^{4}}-\frac{8}{(1-a)}\right) \ln ^{2}(v)+\left(\frac{5}{54 a}-\frac{1}{36 a^{3}}-\frac{43 a}{54\left(1-a^{2}\right)}\right) \ln (v),
\end{aligned}
$$

with

$$
h(a)=\ln ^{2}\left(\frac{1+a}{2}\right)-\frac{1}{\varepsilon} \ln (v)-\zeta(2)+2 \operatorname{Li}_{2}\left(\frac{1+a}{2}\right) .
$$

In the considered case it is possible to obtain an explicit result in terms of hypergeometric functions and hence to perform the $\varepsilon$ expansion using directly the exact formulae. However, from our experience with the $\varepsilon$ expansion at $d=4-2 \varepsilon$, we may say that this is a quite formidable and errorprone task. Thus, having done it once, it is much simpler to use the $d$ recurrence relations in order to get the result in the other dimension. In cases when the analytic result is not known, it might be useful to choose the most convenient $d$ from the point of view of the $\varepsilon$ expansion and then to transform the result to the required space-time dimension. Especially this procedure may be helpful if one encounters integrals with infrared as well as ultraviolet divergences.

\section{CONCLUSIONS}

We proposed a new type of recurrence relations for Feynman integrals. The addition of the space-time dimension to the set of recurrence parameters for Feynman integrals is quite natural and it extends the set of recurrence relations obtained from the integration by parts method. We expect that within the framework of the extended set of recurrence relations it will be possible to formulate rather effective algorithms for the computation of Feynman diagrams. The problem with irreducible numerators in Feynman integrals finds a natural interpretation in the frame of generalized re- currence relations. We demonstrated that a direct solution of the new recurrence relations is possible. Finding solutions of such relations would considerably simplify if it would be possible to develop an effective algorithm for the asymptotic expansion at $|d| \rightarrow \infty$. At the same time the expansion at $|d| \rightarrow \infty$ could be used as an approximation for the integral at finite $d$. This kind of expansion is well known in solid state and statistical physics. Basically, in the present publication we formulated general ideas about new techniques which we intend to apply later to the calculation of some particular classes of Feynman integrals.

\section{ACKNOWLEDGMENTS}

I am grateful to D.V. Shirkov and S.V. Mikhailov for their proposal to perform the evaluation of the integrals discussed in Sec. VI, which became a starting point for the present investigation. I am also grateful to J. Fleischer and F. Jegerlehner for useful discussions and for carefully reading the manuscript. Financial support from BMBF during my stay at the University of Bielefeld and from the Russian Basic Research Foundation (Grant No. 93-02 14428) is gratefully acknowledged. I want to thank also the Aspen Center for Physics (where part of this work was done) for the warm hospitality and financial support. 
[1] J. Zinn-Justin, Quantum Field Theory and Critical Phenomena, 2nd ed. (Oxford University Press, New York, 1993); A. M. Tsvelik, Quantum Field Theory in Condensed Matter Physics (Cambridge University Press, Cambridge, England, 1995); D. J. Amit, Field Theory, the Renormalization Group and Critical Phenomena (McGraw-Hill, New York, 1978).

[2] G. 't Hooft and M. Veltman, Nucl. Phys. B44, 189 (1972); G. G. Bollini and J. J. Giambiagi, Nuovo Cimento B 12, 20 (1972)

[3] S. A. Larin, F. V. Tkachov, and J. A. M. Vermaseren, Report No. NIKHEF-H/91-18, 1991 (unpublished).

[4] C. M. Bender, S. Boettcher, and L. Lipatov, Phys. Rev. D 46, 5557 (1992).

[5] K. G. Chetyrkin and F. V. Tkachov, Nucl. Phys. B192, 159 (1981); F. V. Tkachov, Phys. Lett. 100B, 65 (1981).

[6] V. Alfaro, B. Jacksič, and T. Regge, High Energy Physics and Elementary Particles (International Atomic Energy Agency, Vienna, 1965), pp. 263-273; T. Regge, Algebraic Topology Methods in the Theory of Feynman Relativistic Amplitudes, Battle Recontres, 1967, Lectures in mathematics and physics (Benjamin, New York, 1968). MR 38, 955.

[7] E. E. Boos and A. I. Davydychev, Teor. Mat. Fiz. 89, 56 (1991) [Theor. Math. Phys. 89, 1052 (1991)].

[8] D. J. Broadhurst, J. Fleischer, and O. V. Tarasov, Z. Phys. C 60, 287 (1993).

[9] S. Bauberger, F. A. Berends, M. Böhm, and M. Buza, Nucl. Phys. B434, 383 (1995).

[10] O. V. Tarasov, Nucl. Phys. B (to be published).
[11] C. Itzykson and J.-B. Zuber, Quantum Field Theory (McGrawHill, New York, 1980).

[12] E. R. Speer, J. Math. Phys. (N.Y.) 15, 1 (1974); M. C. Bergère and Yuk-Ming P. Lam, Commun. Math. Phys. 39, 1 (1974).

[13] N. N. Bogoliubov and D. V. Shirkov, Introduction to the Theory of Quantized Fields (Wiley, New York, 1980).

[14] A. I. Davydychev, Phys. Lett. B 263, 107 (1991).

[15] O. V. Tarasov, "The construction of renormalized coefficient functions of Feynman diagrams by computer," Report No. JINR, E2-11573, Dubna, 1978 (unpublished).

[16] A. I. Davydychev and B. Tausk, Phys. Rev. D 53, 7381 (1996).

[17] D. J. Broadhurst, Z. Phys. C 54, 599 (1992).

[18] L. Avdeev, J. Fleischer, S. Mikhailov, and O. Tarasov, Phys. Lett. B 336, 560 (1994); 349, 597(E) (1995).

[19] L. M. Brown, Nuovo Cimento 22, 178 (1961).

[20] D. B. Melrose, Nuovo Cimento A 40, 181 (1965).

[21] F. R. Halpern, Phys. Rev. Lett. 10, 310 (1963).

[22] Z. Bern, L. Dixon, and D. A. Kossover, Nucl. Phys. B412, 751 (1994).

[23] A. J. Macfarlane and G. Woo, Nucl. Phys. B77, 91 (1974); B86, 548(E) (1975); D. I. Kazakov, L. R. Lomidze, N. V. Makhaldiani, and A. A. Vladimirov, "Ultraviolet asymptotics in renormalizable scalar theories," Report No. E2-8085, Dubna, JINR, 1974 (unpublished); A. V. Efremov, V. A. Nesterenko, and A. V. Radyushkin, Nuovo Cimento A 76, 122 (1983). 